\title{
Correlation functions and conditioned quantum dynamics in photodetection theory
}

\author{
Qing Xu, Eliska Greplova, Brian Julsgaard, and Klaus Mølmer \\ Department of Physics and Astronomy, Aarhus University, Ny Munkegade 120, \\ DK-8000 Aarhus C, Denmark \\ E-mail: moelmer@phys.au.dk \\ 30 June 2015
}

\begin{abstract}
Correlations in photodetection signals from quantum light sources are conventionally calculated by application of the source master equation and the quantum regression theorem. In this article we show how the conditioned dynamics, associated with the quantum theory of measurements, allows calculations and offers interpretations of the behaviour of the same quantities. Our theory is illustrated for photon counting and field-amplitude measurements, and we show, in particular, how transient correlations between field-amplitude measurements and later photon counting events can be accounted for by a recently developed theory of past quantum states of a monitored quantum system.
\end{abstract}

\section{Introduction}

The experimental observation in 1956 [1] by Hanbury-Brown and Twiss of temporal correlations in photodetection signals constitutes a defining moment for the field of quantum optics. While the signals measured were compatible with classical field amplitude fluctuations, in a matter of few years it became evident that temporal correlations in signals from quantum light sources, such as a single atom, would defy classical interpretation. Many scientists have since then contributed to these developments and studies of how correlations in photodetection records reveal the quantum properties of the emitter, of the light field, and even of the measurement process itself. Today, optical detection still holds a prominent role in both fundamental tests of quantum theory and in efforts to explore quantum phenomena, e.g., in precision sensing and secure communication protocols.

Correlation functions play an important role in the analysis of time dependent processes with applications ranging from demographics and finance to engineering and physics. Stochastic realizations of different signals can be characterized by, e.g., their mean values and variances while their temporal dynamics is captured by correlations between their values at different times. One may thus evaluate products of two signals at different times, $m_{1}\left(t_{1}\right) m_{2}\left(t_{2}\right)$, and define the temporal (two-time) correlation function 
of the signals as the average of this quantity, $\overline{m_{1}\left(t_{1}\right) m_{2}\left(t_{2}\right)}$, over different realizations of the process. While the expression deals with classical signals $m_{1}(t)$ and $m_{2}(t)$, they may have their origin in measurements on a quantum system, and hence we must apply quantum theory to analyze and make predictions for the signal correlations.

A central position in this research is held by Glauber's photodetection theory [2 4], which explicitly recognized that in the process of light detection, the measurement signal is a fluctuating classical current, $i(t)$, formed by electrons that have been excited by the absorption of photons. The mean value $\overline{i(t)}$ is proportional to the quantum expectation value $\left\langle\hat{a}^{\dagger}(t) \hat{a}(t)\right\rangle$, but to determine the correlation function $\overline{i\left(t_{1}\right) i\left(t_{2}\right)}$ for $t_{1}<t_{2}$, we must incorporate the effect of subsequent individual photon-absorption events, leading to the normal- and time-ordered quantum expectation value $\left\langle\hat{a}^{\dagger}\left(t_{1}\right) \hat{a}^{\dagger}\left(t_{2}\right) \hat{a}\left(t_{2}\right) \hat{a}\left(t_{1}\right)\right\rangle$ of the field creation and annihilation operators.

Glauber's photodetection theory is formulated in the Heisenberg "operator picture" of quantum mechanics, and the evaluation of correlation functions traditionally applies the master equation and the quantum regression theorem for the evaluation of the observables of the light emitting system [5,6]. In this article we show how photodetection theory can also be naturally formulated within the general quantum theory of measurements, which evaluates the the joint probability distribution $P\left(m_{1}, t_{1} ; m_{2}, t_{2}\right)$, that photodetection signals acquire the values $m_{1}\left(t_{1}\right)=m_{1}$ and $m_{2}\left(t_{2}\right)=m_{2}$, by careful evaluation of the back action on the emitter state due to the first measurement and its subsequent evolution until the second one. When one knows the joint probability distribution, the correlation function follows by

$$
\overline{m_{1}\left(t_{1}\right) m_{2}\left(t_{2}\right)}=\sum_{m_{1}, m_{2}}\left(m_{1} m_{2}\right) P\left(m_{1}, t_{1} ; m_{2}, t_{2}\right)
$$

where sums can be interchanged by integrals in the case of continuous spectra of measurement outcomes.

The quantum measurement theory approach accounts explicitly for the back action on the state due to measurements on the system in a manner similar to the stochastic quantum trajectory description [7]. Thus, it both reproduces the results of the quantum regression theorem and sheds light on the origin of the temporal correlations in optical detection records. We demonstrate here that the recent past quantum state formalism [8] provides a similar back action, but on the state prior to a measurement event and thus offers a "symmetric intuition" to the inherently asymmetric case of cross correlations between intensity and amplitude measurements.

The article is organized as follows: In section 2, we introduce the master equation and recall how the quantum regression theorem enables the calculation of photodetection correlation functions. In section 3 we review some aspects of the general quantum theory of measurements that allows an alternative formulation of correlation function calculations, which we extent to the past quantum state formalism in section 4. In section 5, we use this alternative formulation to determine and explain interesting correlation functions between intensity and amplitude measurements of light emitted by two- and three-level atoms, and section 6 concludes the article. 


\section{Correlation functions in photodetection theory}

\subsection{The master equation and the quantum regression theorem}

A light-emitting quantum system can be described by a density matrix $\rho$ which, under the assumption of the Born-Markov approximation, i.e., in the case of weak coupling to memoryless reservoir modes, obeys a linear master equation,

$$
\dot{\rho}=\mathcal{L} \rho,
$$

where

$$
\mathcal{L} \rho=\frac{1}{\mathrm{i} \hbar}[H, \rho]+\sum_{n} \hat{C}_{n} \rho \hat{C}_{n}^{\dagger}-\frac{1}{2}\left(\hat{C}_{n}^{\dagger} \hat{C}_{n} \rho+\rho \hat{C}_{n}^{\dagger} \hat{C}_{n}\right)
$$

$H$ is the Hamiltonian of the system (possibly driven by time-dependent external classical fields) while the operators $\hat{C}_{n}$ account for dissipative couplings to the environment of the system.

If the density matrix elements $\rho_{i j}$ are arranged in a vector, $\mathcal{L}$ can be represented as a matrix, and if this matrix has constant coefficients, equation (2) is formally solved by matrix exponentiation. The propagator of the master equation is a little more complicated than a simple matrix exponential if the equation is time dependent, but we shall nonetheless denote it by the symbol $\mathrm{e}^{\mathcal{L} \tau}$ and use the expression

$$
\rho(t+\tau)=\mathrm{e}^{\mathcal{L} \tau}[\rho(t)]
$$

for the solution to equation (2).

As stated in the Introduction, Glauber's photodetection theory reflects how a detection signal is obtained by absorption processes in the detector. The average product of intensities and amplitudes measured at different times is thus formally related to the expectation value of a normal ordered product of field creation and annihilation operators, $\hat{a}^{\dagger}(t)$ and $\hat{a}\left(t^{\prime}\right)$. The mean intensity at time $t$ is given by $\left\langle\hat{a}^{\dagger}(t) \hat{a}(t)\right\rangle$, while the intensity-intensity correlation function, i.e., the expectation value of the product of intensity measurements at two times $t_{1}<t_{2}$ is $G^{(2)}\left(t_{1}, t_{2}\right)=\left\langle\hat{a}^{\dagger}\left(t_{1}\right) \hat{a}^{\dagger}\left(t_{2}\right) \hat{a}\left(t_{2}\right) \hat{a}\left(t_{1}\right)\right\rangle$, where the superscript (2) refers to the quadratic dependence on intensity.

Like in classical electrodynamics, the electric and magnetic fields can be expressed as functions of the charge and current distributions, and in [9] it is described in detail how the field creation and annihilation operators develop components proportional to the raising and lowering dipole operators of the light emitting system. For a transition in an atom between a definite pair of excited and ground states $|e\rangle$ and $|g\rangle$, the atomic emission of photons with rate $\gamma$ is represented by the operator $\hat{C}=\sqrt{\gamma}|g\rangle\langle e|$ in (3), and hence the relevant field annihilation and creation operators, $\hat{a}$ and $\hat{a}^{\dagger}$, are replaced by the atomic transition operators $\hat{\sigma}=|g\rangle\langle e|$ and $\hat{\sigma}^{\dagger}=|e\rangle\langle g|$. The two-time intensityintensity correlation function is thus proportional to the correlation function of atomic operators,

$$
G^{(2)}\left(t_{1}, t_{2}\right) \propto\left\langle\hat{\sigma}^{\dagger}\left(t_{1}\right) \hat{\sigma}^{\dagger}\left(t_{2}\right) \hat{\sigma}\left(t_{2}\right) \hat{\sigma}\left(t_{1}\right)\right\rangle
$$


The two-time expectation values in (5) is on the form $\left\langle\hat{O}_{1}(t) \hat{O}_{2}(t+\tau) \hat{O}_{3}(t)\right\rangle=$ $\operatorname{Tr}\left(\hat{O}_{1}(t) \hat{O}_{2}(t+\tau) \hat{O}_{3}(t) \rho(t)\right)=\operatorname{Tr}\left(\hat{O}_{2}(t+\tau) \hat{O}_{3}(t) \rho(t) \hat{O}_{1}(t)\right)$, where the central operator $\hat{O}_{2}(t+\tau)\left(=\hat{\sigma}^{\dagger}\left(t_{2}\right) \hat{\sigma}\left(t_{2}\right)\right)$ can be expanded on the complete set of dyadic operators $(|j\rangle\langle i|)(t+\tau)$. The expectation values of these operators are the density matrix elements, $\rho_{i j}=\langle(|j\rangle\langle i|)\rangle$, which obey the linear set of coupled equations defined by the master equation (22). The same is, however, not true for the operators $(|j\rangle\langle i|)$ themselves, and noise operator terms must be added to the equations to ensure the preservation of commutation and uncertainty relations [5, 6]. In the Markov approximation, however, these noise operators are uncorrelated with all system observables at earlier times, and this implies that two- and multi-time operator correlation functions of the form, $\left\langle\hat{O}_{1}(t)(|j\rangle\langle i|)(t+\tau) \hat{O}_{3}(t)\right\rangle$, indeed, evolve with the time argument $\tau$ according to the same linear set of coupled equations as $\langle(|j\rangle\langle i|)(t+\tau)\rangle$, i.e., as $\rho_{i j}(t+\tau)$, as long as $\tau>0$ and $\hat{O}_{1}(t)$ and $\hat{O}_{3}(t)$ represent operators, evaluated at earlier times. This is the Quantum Regression Theorem [6, 10], and it offers an effective means to determine correlation functions of operators for Markovian open quantum system.

For comparison with experiments, we are interested in steady state dynamics, and hence only in the dependence of the correlation functions on the time difference $\tau$. We thus assume that $\rho(t)=\rho^{\text {st }}$, the steady state solution of the master equation, and, for $\tau>0$, the quantum regression theorem yields the formal solution [6, 10]

$$
\left\langle\hat{O}_{1}(t) \hat{O}_{2}(t+\tau) \hat{O}_{3}(t)\right\rangle=\operatorname{Tr}\left(\hat{O}_{2} \mathrm{e}^{\mathcal{L} \tau}\left[\hat{O}_{3} \rho^{\mathrm{st}} \hat{O}_{1}\right]\right) .
$$

The master equation is linear and preserves the trace and hence, for any matrix $\mu$, $\mathrm{e}^{\mathcal{L} \tau}[\mu] \rightarrow \operatorname{Tr}(\mu) \rho^{\text {st }}$ for $\tau \rightarrow \infty$. This implies that the correlation function (6) regresses

from the steady state mean value of the operator product $\left\langle\hat{O}_{1} \hat{O}_{2} \hat{O}_{3}\right\rangle_{\mathrm{st}}$ at $\tau=0$ to the product of steady state mean values $\left\langle\hat{O}_{2}\right\rangle_{\mathrm{st}}\left\langle\hat{O}_{1} \hat{O}_{3}\right\rangle_{\mathrm{st}}$. In quantum optics it is convenient to normalize the correlation functions by this product of the steady state mean values. The resulting reduced correlation functions, denoted by a lower case letter $g$, then approach unity for large $\tau$.

\subsection{Intensity-intensity and intensity-amplitude correlations}

With the choice of operators $\hat{O}_{1}=\hat{\sigma}^{\dagger}, \hat{O}_{3}=\hat{\sigma}$, and $\hat{O}_{2}=\hat{\sigma}^{\dagger} \hat{\sigma}$, the intensity-intensity correlation function $G^{(2)}(\tau)$ (5) is given by (6). With these operators, $\hat{O}_{3} \rho^{\text {st }} \hat{O}_{1}=$ $\rho_{e e}^{\text {st }}|g\rangle\langle g|$, i.e., the atomic ground state density operator, multiplied by the steady state excitation probability $\rho_{e e}^{\text {st }}$. In this case, the evolution by $\exp (\mathcal{L} \tau)$ merely solves the transient evolution of the atomic density matrix, starting from the ground state, and since $\hat{O}_{2}=|e\rangle\langle e|$ is the projection operator on the excited state, the reduced correlation function evaluates to $g^{(2)}(\tau)=\left.\rho_{e e}^{\text {st }} \rho_{e e}(\tau)\right|_{g} /\left(\rho_{e e}^{\text {st }}\right)^{2}=\left.\rho_{e e}(\tau)\right|_{g} / \rho_{e e}^{\text {st }}$, where $\left.\rho_{e e}(\tau)\right|_{g}$ denotes the excited state population of a system, evolved from the ground state at $\tau=0$. This result is readily understood from the quantum trajectory picture [7,11,12]: The photon detection at time $t$ is accompanied by an atomic quantum jump into the ground state, and the probability to register another photon from the same atom at the later time $t+\tau$ is proportional to the excited state population conditioned on the first detection. 


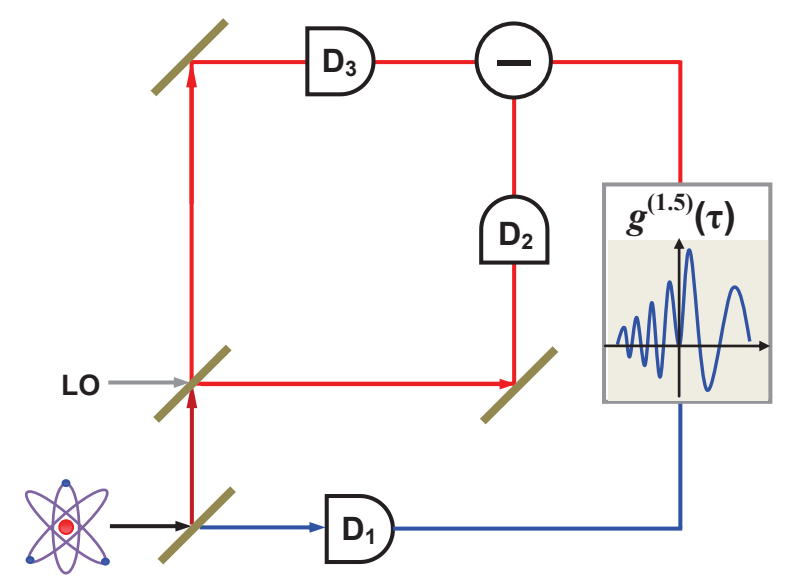

Figure 1. A setup for joint photon counting and homodyne detection. Light transmitted by the beam splitter along the lower path is detected as discrete photon counting events, while the light reflected along the upper path is mixed with a strong local oscillator and the difference between the two counting signals represents the continuous homodyne monitoring of the emitted field amplitude. Averaging the noisy, time dependent homodyne signal in time windows around all counting events in the detector $\mathrm{D}_{1}$, we obtain the amplitude-intensity correlation function.

The particle and the wave description of optical phenomena competed as the dominant descriptions of light until Planck and Einstein introduced the dual quantum nature of light more than a century ago. In beautiful, more recent demonstrations of the joint wave and particle properties of light [13, 14, the radiation emitted by an atom was split in two components, see figure 1, for which the photon number and the field amplitude were detected, respectively. These experiments revealed strong temporal correlations between the measurement outcomes. For a positive time delay, $\tau>0$, between the counting events and the amplitude measurement, such amplitude-intensity correlations are captured by the time- and normal-ordered coherence function [13]

$$
g^{(1.5)}(\tau>0)=\frac{\left\langle\hat{\sigma}^{\dagger}(t)\left(\mathrm{e}^{\mathrm{i} \varphi} \hat{\sigma}^{\dagger}(t+\tau)+\mathrm{e}^{-\mathrm{i} \varphi} \hat{\sigma}(t+\tau)\right) \hat{\sigma}(t)\right\rangle}{\rho_{e e}^{\mathrm{st}}\left(\mathrm{e}^{\mathrm{i} \varphi} \rho_{g e}^{\mathrm{st}}+\mathrm{e}^{-\mathrm{i} \varphi} \rho_{e g}^{\mathrm{st}}\right)},
$$

where $\varphi$ is the phase component measured by the homodyne detector, and where the superscript (1.5) refers to the "intensity ${ }^{1.5 "}$ "-dependence of the function. The numerator in this expression is represented by (6), with $\hat{O}_{1}=\hat{\sigma}^{\dagger}, \hat{O}_{3}=\hat{\sigma}$, and $\hat{O}_{2}=\mathrm{e}^{\mathrm{i} \varphi} \hat{\sigma}^{\dagger}+\mathrm{e}^{-\mathrm{i} \varphi} \hat{\sigma}$, and its behaviour can be understood from the quantum jump of the steady state density matrix into the ground state at time $t$, and the evaluation of the expectation value of the amplitude operator $\hat{O}_{2}$ during the subsequent transient evolution of the conditional state.

In the experiments [13, 14, the homodyne amplitude signal shows correlations also with later photon counting events. The measured correlations involve the same operators $\hat{O}_{i}$ as before, but for $\tau<0$, the time- and normal-ordering procedure leads instead to 


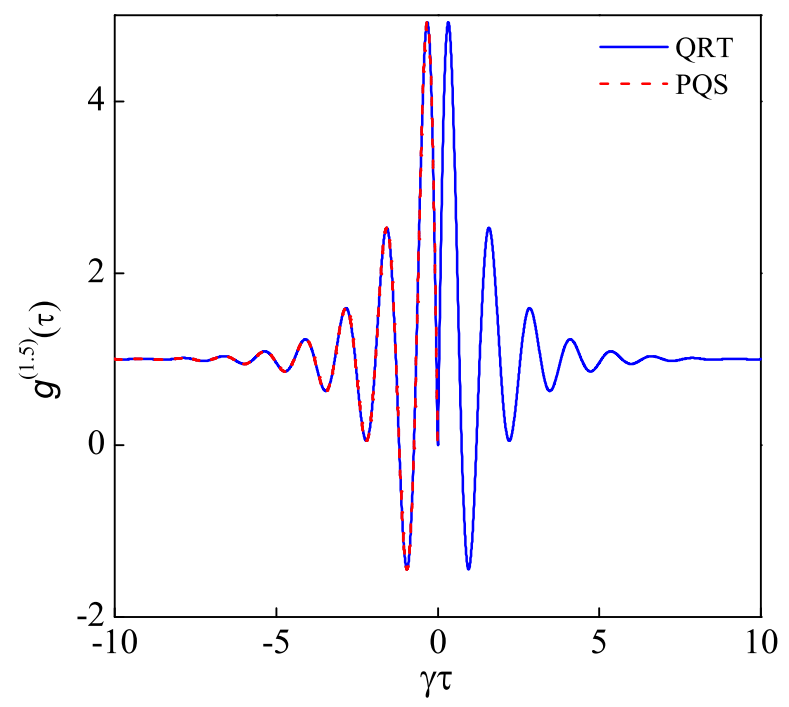

Figure 2. Amplitude-intensity correlation function (7) and (8) for a resonantly driven two-level atom determined by the quantum regression theorem (blue solid line) and by the past quantum state (red dashed line) (19). The parameters are: $\Omega=5 \gamma$ and $\varphi=\pi / 2$.

the expression [15]

$$
g^{(1.5)}(\tau<0)=\frac{\mathrm{e}^{\mathrm{i} \varphi}\left\langle\hat{\sigma}^{\dagger}(t) \hat{\sigma}^{\dagger}(t-\tau) \hat{\sigma}(t-\tau)\right\rangle+\mathrm{e}^{-\mathrm{i} \varphi}\left\langle\hat{\sigma}^{\dagger}(t-\tau) \hat{\sigma}(t-\tau) \hat{\sigma}(t)\right\rangle}{\rho_{e e}^{\mathrm{st}}\left(\mathrm{e}^{\mathrm{i} \varphi} \rho_{g e}^{\mathrm{st}}+\mathrm{e}^{-\mathrm{i} \varphi} \rho_{e g}^{\mathrm{st}}\right)} .
$$

In the evaluation of (8), the time evolution operator in (6) is applied to products of matrices $\rho^{\text {st }} \hat{\sigma}^{\dagger}$ and $\hat{\sigma} \rho^{\text {st }}$ that do not permit separate interpretation as quantum states, and unlike the case of $\tau>0$, we do not have a simple interpretation of the expression in (8) in terms of the transient conditioned dynamics of the system. Wiseman has shown [16], however, that the weak value formalism [17] for the post selected average of a weakly perturbing measurement, indeed, accounts for field-amplitude correlations with later count events. That formalism is related to the recently developed theory of past quantum states [8], and as described below, the general quantum theory of measurements and the past quantum state, allow both calculation (the red dashed curve in figure 2) and interpretation of how field-amplitude measurements are correlated with later detector click event.

In figure 2, we show the value of the normalized correlation function $g^{(1.5)}(\tau)$ for a two-level atom for both positive and negative $\tau$, and it is interesting that despite the very different formal expressions, the evaluation of (7) and (8) yields a symmetric correlation function around $\tau=0$. Since intensity-intensity correlation functions concern fully equivalent detection events, they have to be symmetric, but as we shall see below, intensity-amplitude correlation functions may, indeed, be very asymmetric. 


\section{Quantum measurement theory and field correlations}

Equation (2) describes the average behavior of an unobserved light emitting system, while observation of the emitted radiation will yield a conditioned dynamics, described by the general theory of measurements. Formally, any observation of a quantum system is described by a positive operator valued measure (POVM), i.e., a set of operators $\hat{\Omega}_{m}$, with $\sum_{m} \hat{\Omega}_{m}^{\dagger} \hat{\Omega}_{m}=I$, where $I$ is the identity matrix [12,18]. The operators $\hat{\Omega}_{m}$ account for the joint evolution of the system and an appropriate meter system and read-out of the meter in the states that we associate with the outcome results $m$. The probability to get a definite outcome $m$ is given by the expression,

$$
P(m)=\operatorname{Tr}\left(\rho \hat{\Omega}_{m}^{\dagger} \hat{\Omega}_{m}\right),
$$

and the (normalized) state of the system, conditioned on this measurement outcome, is

$$
\rho_{m}=\hat{\Omega}_{m} \rho \hat{\Omega}_{m}^{\dagger} / P(m) .
$$

If $\left\{\hat{\Omega}_{m}\right\}$ constitutes a complete set of orthogonal projection operators, the POVM formalism yields the conventional Born rule and von Neumann projection postulate.

The POVM formalism also gives access to the probability that a measurement, described by POVM operators $\hat{\Omega}_{m}$ yields $m_{1}$ at time $t_{1}$ and a subsequent measurement at $t_{2}$ on the same system, described by the same or a different set of POVM operators, yields the outcome $m_{2}$. Such joint probabilities follow Bayes' rule, and can be written as

$$
P\left(m_{1}, t_{1} ; m_{2}, t_{2}\right)=P\left(m_{1}, t_{1}\right) \cdot P\left(m_{2}, t_{2} \mid m_{1}, t_{1}\right) .
$$

Assuming steady state conditions, the unconditional probability $P\left(m_{1}\right)=\operatorname{Tr}\left(\hat{\Omega}_{m_{1}} \rho^{\text {st }} \hat{\Omega}_{m_{1}}^{\dagger}\right)$ for the first event to occur does not depend on the time argument $t_{1}$, and the state of the system, conditioned on this outcome is $\rho_{m_{1}}\left(t_{1}\right)=\hat{\Omega}_{m_{1}} \rho^{\text {st }} \hat{\Omega}_{m_{1}}^{\dagger} / P\left(m_{1}\right)$. Propagating the density matrix further, until $t_{2}$, by the usual master equation with $\rho_{m_{1}}\left(t_{1}\right)$ as the initial condition, we find

$$
\rho_{m_{1}}\left(t_{2}\right)=\mathrm{e}^{\mathcal{L}\left(t_{2}-t_{1}\right)} \rho_{m_{1}}\left(t_{1}\right) .
$$

For the joint outcome probability distribution, we thus obtain

$$
P\left(m_{1}, t_{1} ; m_{2}, t_{2}\right)=\operatorname{Tr}\left(\hat{\Omega}_{m_{2}} \mathrm{e}^{\mathcal{L}\left(t_{2}-t_{1}\right)}\left[\hat{\Omega}_{m_{1}} \rho^{\mathrm{st}} \hat{\Omega}_{m_{1}}^{\dagger}\right] \hat{\Omega}_{m_{2}}^{\dagger}\right),
$$

where $\mathrm{e}^{\mathcal{L}\left(t_{2}-t_{1}\right)}$ acts on the matrix object contained within the square brackets.

Equation (13) provides the outcome joint probabilities and offers a microscopic, dynamic view of a conditionally evolved quantum state. This differs from (6) which directly provides the average correlation function of the physical observables, e.g., of the field and intensity operators. They are connected, however, by using equation (13) for the calculation of weighted mean values of the outcomes: If the labels $m_{1}$ and $m_{2}$ denote the actual values of the physical observables measured at times $t_{1}$ and $t_{2}$, the operator correlation function of interest acquires the form (1),

$$
\overline{m_{1} m_{2}}=\sum_{m_{1}, m_{2}}\left(m_{1} m_{2}\right) P\left(m_{1}, t_{1} ; m_{2}, t_{2}\right) .
$$




\subsection{Photon counting and homodyne detection}

Restricting ourselves to the case of detection of light emitted from a quantum system, photon counting associates two POVM operators to the click and no-click detector events during an infinitesimal time interval $\mathrm{d} t$. The observation of a photon from a two-level atom is described by $\hat{\Omega}_{1}=\sqrt{\gamma \mathrm{d} t} \hat{\sigma}$, while the absence of a detector click is described, to first order in $\mathrm{d} t$, by $\hat{\Omega}_{0}=\left(I-\frac{\gamma \mathrm{d} t}{2} \hat{\sigma}^{\dagger} \hat{\sigma}\right)$, reproducing the respective probabilities $\rho_{e e} \gamma \mathrm{d} t$ and $1-\rho_{e e} \gamma \mathrm{d} t$ as well as the final atomic states after the detection events. The atomic states are notably not orthogonal for the two field detection outcomes. The average behavior of the system, $\rho \rightarrow \sum_{m} \hat{\Omega}_{m} \rho \hat{\Omega}_{m}^{\dagger}$, subject to the two outcome possibilities is exactly the one described by the damping terms in the master equation (2) with a single $\hat{C}=\sqrt{\gamma} \hat{\sigma}$.

In homodyne detection, the light signal is mixed with a strong classical field of phase $\varphi$, and the intensity of the interfering fields is recorded by two photon counters, as shown in figure 1. The difference between their counting signals is represented by a continuous quantity $x$, which in the absence of the emitter is represented by a Gaussian distribution, $P_{0}(x)=\frac{1}{\sqrt{2 \pi / \mathrm{d} t}} \exp \left(-x^{2} \mathrm{~d} t / 2\right)$, with a suitable normalization of the outcome argument $x$ [12, 16]. In the presence of the two-state emitter, the outcome is governed by the family of POVM operators,

$$
\hat{\Omega}_{x}=\left(\frac{\mathrm{d} t}{2 \pi}\right)^{\frac{1}{4}} \exp \left(-\frac{x^{2}}{4} \mathrm{~d} t\right)\left(1+x \mathrm{~d} t \sqrt{\gamma} \mathrm{e}^{-\mathrm{i} \varphi} \hat{\sigma}-\frac{\gamma \mathrm{d} t}{2} \hat{\sigma}^{\dagger} \hat{\sigma}\right) .
$$

To first order in $\mathrm{d} t$, one readily verifies the POVM property $\int \hat{\Omega}_{x}^{\dagger} \hat{\Omega}_{x} \mathrm{~d} x=I$ and that the average change of the state, $\rho \rightarrow \int \hat{\Omega}_{x} \rho \hat{\Omega}_{x}^{\dagger} \mathrm{d} x$, reproduces the effect of the damping term $\hat{C}=\sqrt{\gamma} \hat{\sigma}$ in (3)).

The mean intensity measured as the average number of detection events per time, $\bar{n} / d t$, has an expectation value given by the weighted mean of the possible outcomes of the photon counting measurement, $\bar{n}=\sum_{n=0,1} n \cdot P(n)=\sum_{n=0,1} n \cdot \operatorname{Tr}\left(\hat{\Omega}_{n} \rho \hat{\Omega}_{n}^{\dagger}\right)=\rho_{e e}^{\text {st }} \gamma \mathrm{d} t$, where the last expression applies for the two-level atom in steady state prior to the measurement. Similarly, in homodyne detection, the one-time mean amplitude of the field is proportional to $\langle x\rangle=\int x \cdot P(x) \mathrm{d} x=\int x \cdot \operatorname{Tr}\left(\hat{\Omega}_{x} \rho \hat{\Omega}_{x}^{\dagger}\right) \mathrm{d} x=\sqrt{\gamma} \operatorname{Tr}\left(\mathrm{e}^{-\mathrm{i} \varphi} \hat{\sigma} \rho+\right.$ $\left.\mathrm{e}^{\mathrm{i} \varphi} \rho \hat{\sigma}^{\dagger}\right)=\sqrt{\gamma}\left(\mathrm{e}^{-\mathrm{i} \varphi} \rho_{e g}^{\mathrm{st}}+\mathrm{e}^{\mathrm{i} \varphi} \rho_{g e}^{\mathrm{st}}\right)$.

The two-time intensity-intensity correlation function is proportional to $\sum_{n_{1}, n_{2}=0,1} n_{1} n_{2} P\left(n_{1}, t_{1} ; n_{2}, t_{2}\right)=P\left(n_{1}=1, t_{1} ; n_{2}=1, t_{2}\right)$ with both of the POVM operators in equation (13) pertaining to photon counting. Applying the expressions for a two-level emitter, we thus obtain $P\left(n_{1}=1, t_{1} ; n_{2}=1, t_{2}=t_{1}+\tau\right)=\left.(\gamma \mathrm{d} t)^{2} \rho_{e e}^{\mathrm{st}} \rho_{e e}(\tau)\right|_{g}$, which is proportional to $G^{(2)}(\tau>0)$, identified in the previous section. In exactly the same way, we can determine the probability for the joint detection of a photon at time $t_{1}$ and the subsequent amplitude outcome $x$ at time $t_{2}, P\left(n, t_{1} ; x, t_{2}\right)$, and we can determine the average $\sum_{n} \int n x P\left(n, t_{1} ; x, t_{2}\right) \mathrm{d} x$. For positive time lag $\tau=t_{2}-t_{1}$ this yields $\rho_{e e}^{\mathrm{st}}\left(\left.\mathrm{e}^{-\mathrm{i} \varphi} \rho_{e g}(\tau)\right|_{g}+\left.\mathrm{e}^{\mathrm{i} \varphi} \rho_{g e}(\tau)\right|_{g}\right) \gamma \sqrt{\gamma} \mathrm{d} t$ which is indeed proportional to $G^{(1.5)}(\tau)$ (77). 


\section{Amplitude-intensity correlations and past quantum state}

We shall now address the joint probability of a homodyne detector outcome $x$ at the earlier time $t_{1}$ followed by the counting of a photon at the later time $t_{2}$, i.e., we take $\hat{\Omega}_{m_{1}}=\hat{\Omega}_{x}$ and $\hat{\Omega}_{m_{2}}=\hat{\Omega}_{n}$ in (13),

$$
\begin{aligned}
P\left(x, t_{1} ; n, t_{2}\right) & =P\left(x, t_{1}\right) \cdot P\left(n, t_{2} \mid x, t_{1}\right) \\
& =\operatorname{Tr}\left(\hat{\Omega}_{n} \mathrm{e}^{\mathcal{L}\left(t_{2}-t_{1}\right)}\left[\hat{\Omega}_{x} \rho^{\mathrm{st}} \hat{\Omega}_{x}^{\dagger}\right] \hat{\Omega}_{n}^{\dagger}\right) .
\end{aligned}
$$

To evaluate the amplitude-intensity correlation function, we multiply the probability by $n$ and $x$, and we sum/integrate over their possible values. This will retain only the $n=1$ component and the contributions that are first order in $x$ in the expressions for $\hat{\Omega}_{x}^{(\dagger)}$. Thus, even though the $\hat{\Omega}_{x}$ operators differ only infinitesimally from the identity and the back action of the $x$ measurement is weak, when we calculate the integral over $x$, only the terms $\propto d t \sqrt{\gamma} e^{-i \varphi} \hat{\sigma}$ and $d t \sqrt{\gamma} e^{i \varphi} \hat{\sigma}^{\dagger}$, cf. (15), contribute to the correlation function average over the very nosy homodyne signal.

Rather than merely reproducing existing results with a different method, the purpose of this work is to analyze the problem and gain new insight into the results. We thus rewrite equation (16) for the joint probability of the outcomes, as $P\left(n, t_{2}\right)$. $P\left(x, t_{1} \mid n, t_{2}\right)$ to emphasize the conditioning on the later photon counting rather than the earlier homodyne detection event. Indeed, such retrodictive conditioning can be applied to any type of measurements, $c f$. the past quantum state formalism [8], applied in [19,20]. We shall briefly recall this formalism before specializing to the calculation and interpretation of amplitude-intensity correlations.

The density matrix evolves according to a completely positive map, and hence, for any operator $\mu$, we can formally write the action of the propagator of the master equation as a Kraus map [12, 18], $\mathrm{e}^{\mathcal{L}\left(t_{2}-t_{1}\right)}[\mu]=\sum_{\alpha} K_{\alpha} \mu K_{\alpha}^{\dagger}$, where $\sum_{\alpha} K_{\alpha}^{\dagger} K_{\alpha}=I$. The operators $K_{\alpha}$ represent the unobserved time evolution between $t_{1}$ and $t_{2}$, and they depend of course on the explicit form of the master equation and the propagation time, but for our purpose we shall not need their explicit form. The cyclic properties of the trace allows us to transform equation (13) into

$$
P\left(m_{1}, t_{1} ; m_{2}, t_{2}\right)=\operatorname{Tr}\left(\hat{\Omega}_{m_{1}} \rho^{\mathrm{st}} \hat{\Omega}_{m_{1}}^{\dagger} \sum_{\alpha} K_{\alpha}^{\dagger} \hat{\Omega}_{m_{2}}^{\dagger} \hat{\Omega}_{m_{2}} K_{\alpha}\right) .
$$

It is now natural to define the operator product $E\left(t_{1}\right) \equiv \sum_{\alpha} K_{\alpha}^{\dagger} \hat{\Omega}_{m_{2}}^{\dagger} \hat{\Omega}_{m_{2}} K_{\alpha}$. The order of the operators $K_{\alpha}^{\dagger}$ and $K_{\alpha}$ has been swapped compared to their action on the time dependent density matrix, and the continuous equation of evolution for $E(t)$ is similarly obtained by swapping the Lindblad operator terms (3) in the master equation (2),

$$
\frac{\mathrm{d} E(t)}{\mathrm{d}(-t)}=\frac{\mathrm{i}}{\hbar}[H, E]+\sum_{n} \hat{C}_{n}^{\dagger} E \hat{C}_{n}-\frac{1}{2}\left(\hat{C}_{n}^{\dagger} \hat{C}_{n} E+E \hat{C}_{n}^{\dagger} \hat{C}_{n}\right) .
$$

We solve the equation for $E(t)$ backwards in time from $t_{2}$ to $t_{1}$ with the boundary condition, $E\left(t_{2}\right)=\hat{\Omega}_{m_{2}}^{\dagger} \hat{\Omega}_{m_{2}}$.

The correlations in the joint probability distribution imply that knowledge of the later measurement outcomes alters the conditional probability for the earlier ones. We 
can formally express this by $P\left(m_{1}, t_{1} ; m_{2}, t_{2}\right)=P\left(m_{2}, t_{2}\right) \cdot P\left(m_{1}, t_{1} \mid m_{2}, t_{2}\right)$. We now determine the first factor $P\left(m_{2}, t_{2}\right)$ by summation over the index $m_{1}$ in (17) and the conditional probability then acquires the form

$$
P\left(m_{1}, t_{1} \mid m_{2}, t_{2}\right)=\frac{\operatorname{Tr}\left(\hat{\Omega}_{m_{1}} \rho\left(t_{1}\right) \hat{\Omega}_{m_{1}}^{\dagger} E\left(t_{1}\right)\right)}{\sum_{m} \operatorname{Tr}\left(\hat{\Omega}_{m} \rho\left(t_{1}\right) \hat{\Omega}_{m}^{\dagger} E\left(t_{1}\right)\right)},
$$

where the matrix $E\left(t_{1}\right)$ and the density matrix $\rho\left(t_{1}\right)$ play symmetric roles, representing how the outcome probabilities are correlated with the prior and the posterior dynamics and measurements of the system, respectively [8]. We are interested in steady state correlation functions, and since we eventually want to account for only the two-time correlation with a single later photon detection event, $\rho\left(t_{1}\right)=\rho^{\text {st }}$. When we solve equation (18), we obtain predictions in full agreement with the quantum regression theorem results.

As $E(t)$ solves an equation which is similar to the master equation for $\rho(t)$, we can exploit our intuition for density matrix evolution and infer that for the two-level atom, $E(t)$ performs an evolution backward in time, starting from the excited state, which is similar to damped Rabi oscillations of the conventional density matrix. This, indeed, gives a qualitative explanation of the red dashed curve in figure 2. The evolution of $E(t)$ involves population loss out of the excited state by the Lindblad damping term $-\frac{1}{2}\left(\hat{C}^{\dagger} \hat{C} E+E \hat{C}^{\dagger} \hat{C}\right)$ with $\hat{C}=\sqrt{\gamma} \hat{\sigma}$, but also population feeding into the excited state by $\hat{C}^{\dagger} E \hat{C}$. Unlike the master equation (21), equation (18) does not preserve the trace, and unlike the density matrix converging towards its steady state value, $\rho^{\text {st }}, E(t)$ converges towards the identity matrix for long (negative) propagation times. It is thus not evident that the correlation function in figure 2 should be completely symmetric in the time argument, but it follows by a closer inspection (and solution) of the equations of evolution.

\section{Amplitude-intensity correlations from three-level atoms}

We shall in this section study the fluorescence from optical transitions in three-level atoms. These systems have alrady been analyzed [13 15, 21, 22], by the quantum regression theorem, and they show a number of features, that we can explain with our theory.

\subsection{Ladder system}

Let us consider a three-level ladder atom with energy levels as drawn in figure 3(a). Its Hamiltonian can be written as

$$
H=-\frac{\hbar}{2}\left(\Omega_{1} \hat{\sigma}_{21}+\Omega_{2} \hat{\sigma}_{32}+\text { H.c. }\right) \text {, }
$$

where $\hat{\sigma}_{i j}=|i\rangle\langle j|,(i, j=1-3), \Omega_{1,2}$ are the (real) laser Rabi frequencies. Quantum

jump operators of (3) are $\hat{C}_{1}=\sqrt{\gamma_{1}} \hat{\sigma}_{12}$ and $\hat{C}_{2}=\sqrt{\gamma_{2}} \hat{\sigma}_{23}$ with decay rates $\gamma_{1,2}$, and 


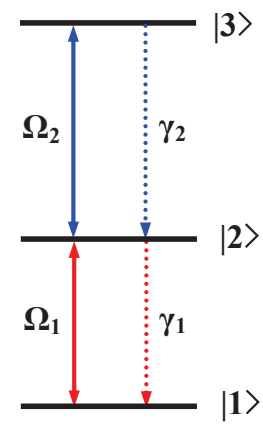

(a)

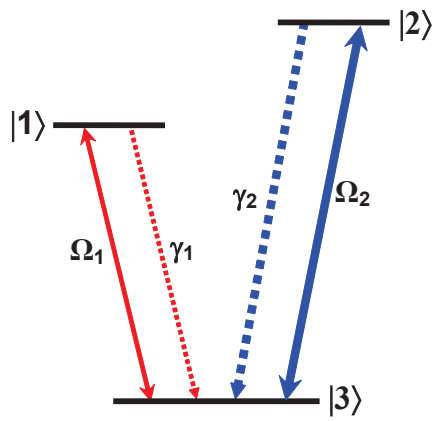

(b)

Figure 3. A three-level atom driven by two resonant laser fields. In the ladder configuration (a), the fields are of comparable strength while the upper state $|3\rangle$ has longer lifetime than the intermediate state $|2\rangle$. In the $V$-configuration (b), the driving field and the decay rate on the $|2\rangle \leftrightarrow|3\rangle$ transition are much stronger than those on the $|1\rangle \leftrightarrow|3\rangle$ transition.

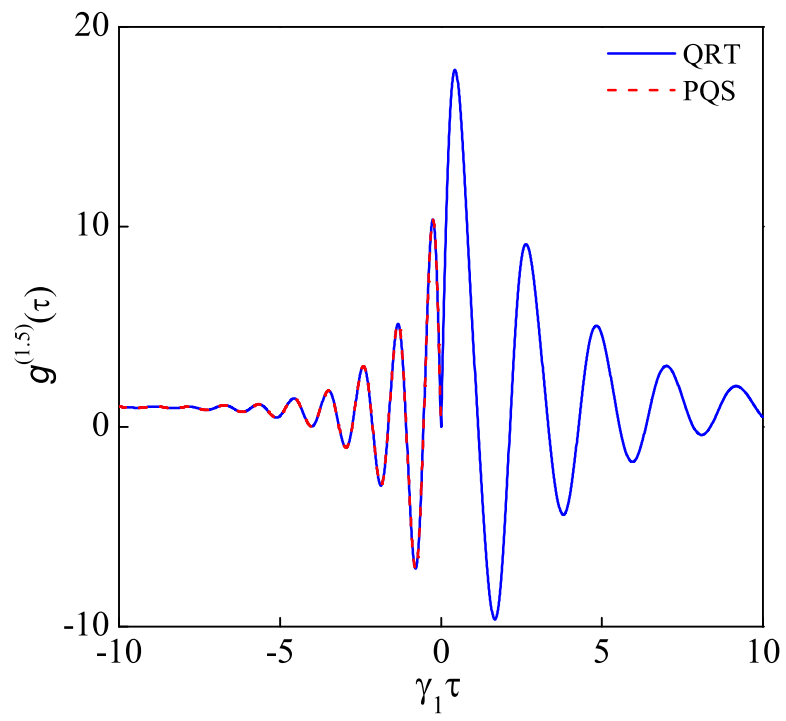

Figure 4. (Color online) Amplitude-intensity correlation on the lower transition of a single three-level ladder atom by invoking the quantum regression theorem (blue solid line) and past quantum state (red dashed line). The parameters are: $\Omega_{1}=3 \gamma_{1}$, $\Omega_{2}=5 \gamma_{1}, \gamma_{2}=0.2 \gamma_{1}, \gamma_{\mathrm{ph}}=5 \times 10^{-5} \gamma_{1}$, and $\varphi=\pi / 2$.

$\hat{C}_{3}=\sqrt{\gamma_{\mathrm{ph}}}\left(\hat{\sigma}_{33}-\hat{\sigma}_{22}-\hat{\sigma}_{11}\right)$ with a dephasing rate $\gamma_{\mathrm{ph}}$ of the upper state with respect to the two lower states.

We have calculated the correlation function $g^{(1.5)}$ between the intensity and amplitude signals in the frequency range of both the lower and the upper transition in the system. The quantum regression theorem, the POVM formulation, and the past quantum state formalism naturally all give the same quantitative predictions for this correlation function, shown in figure 4. 
Unlike the two-level atom, the three-level atomic system shows a striking temporal asymmetry of the correlation function [15]. In particular, for the lower transition the frequency of the correlation function oscillations for the negative time delay is twice the one for positive time delay (see figure 4). The past quantum state reasoning explains why this happens. For $\tau>0$, the photon counting event makes the atom density matrix evolve forwards in time from the ground state $|1\rangle$ at time $t$. For $\tau<0$, the field amplitude can be calculated from the matrix $E$, evolving backwards in time from the excited state of the monitored transition, i.e., the intermediate state $|2\rangle$ in the ladder configuration. In equations (3) and (18) the evolution due to the Hamiltonian is the same (modulo a sign), and if we disregard the dissipative terms, the evolution is readily analyzed in terms of the Hamiltonian eigenstates,

$$
\begin{aligned}
& |+\rangle=\frac{1}{\sqrt{2}}(\cos \theta|1\rangle-|2\rangle+\sin \theta|3\rangle), \\
& |0\rangle=-\sin \theta|1\rangle+\cos \theta|3\rangle, \\
& |-\rangle=\frac{1}{\sqrt{2}}(\cos \theta|1\rangle+|2\rangle+\sin \theta|3\rangle),
\end{aligned}
$$

with corresponding eigenenergies

$$
\mathcal{E}_{+}=\frac{\hbar}{2} \Omega_{\mathrm{R}}, \quad \mathcal{E}_{0}=0, \quad \mathcal{E}_{-}=-\frac{\hbar}{2} \Omega_{\mathrm{R}},
$$

where $\Omega_{\mathrm{R}}=\sqrt{\Omega_{1}^{2}+\Omega_{2}^{2}}$ and $\tan \theta=\frac{\Omega_{2}}{\Omega_{1}}$.

After a photon counting event, the conditioned evolution starts with the atom in state $|1\rangle$, which is a superposition of all three dressed eigenstates. It subsequently evolves with relative phase factors that oscillate with the spectral separation $\Omega_{R} / 2$, which then yields the oscillation frequency of the density matrix elements. The backward evolution of $E(t)$, on the other hand, starts from the state $|2\rangle$, which can be expanded as a superposition of only the $|+\rangle$ and $|-\rangle$ eigenstates which are separated by $\Omega_{R}$. The matrix elements of $E(t)$ thus evolve with a relative frequency of $\Omega_{\mathrm{R}}$, which therefore governs the twice faster oscillations of the two-time correlation function for negative $\tau$.

\subsection{V-system}

The Hamiltonian of the $V$-configuration depicted in figure 3(b) reads

$$
H=-\frac{\hbar}{2}\left(\Omega_{1} \hat{\sigma}_{13}+\Omega_{2} \hat{\sigma}_{23}+\text { H.c. }\right),
$$

and the atom is subject to two quantum jump operators $\hat{C}_{1}=\sqrt{\gamma_{1}} \hat{\sigma}_{31}$ and $\hat{C}_{2}=\sqrt{\gamma_{2}} \hat{\sigma}_{32}$.

As shown in figure 5, the amplitude-intensity correlation function on the weak transition $|1\rangle \leftrightarrow|3\rangle$ in a $V$-system is asymmetric. For $\tau>0$, we observe damped dipole moment oscillations, with an additional modulation that we ascribe to the rapid dynamics on the stronger atomic transition. For $\tau<0$, the oscillations are more regular, however they are damped towards a large almost constant value, and only on a much longer time scale (see insert), the correlation function converges to unity. We can account 


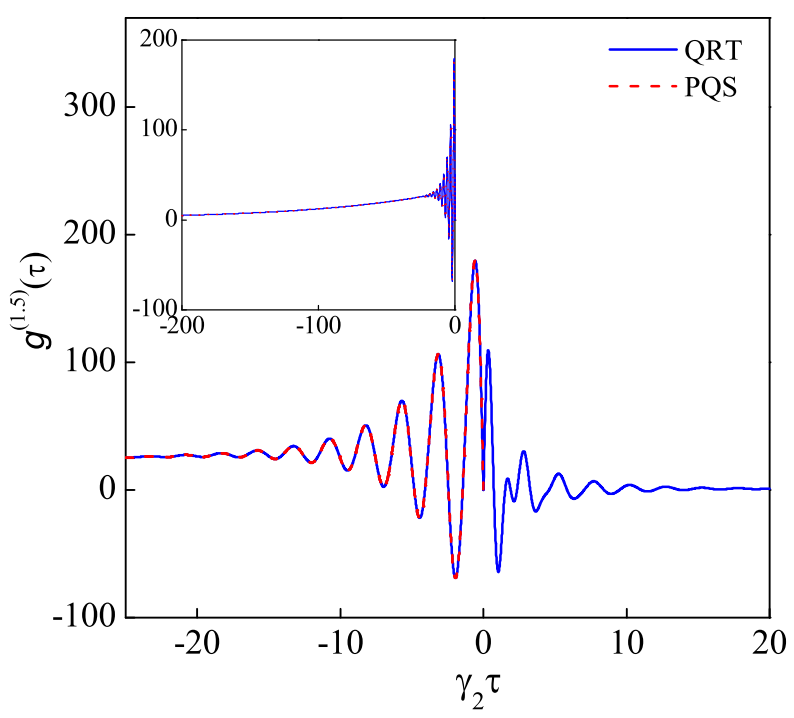

Figure 5. Amplitude-intensity correlation on the weak probe transition of a single three-level $V$ atom by invoking the quantum regression theorem (blue solid line) and past quantum state (red dashed line). The inset indicates a long (negative) time trace approaching unity on the "Zeno time scale". The parameters are: $\Omega_{1}=0.1 \gamma_{2}$, $\Omega_{2}=5 \gamma_{2}, \gamma_{1}=0.01 \gamma_{2}$, and $\varphi=\pi / 2$.

for both of these observations by the backward evolution of the matrix $E(t)$ : First, we note that $E(t)$ has the excited state $|1\rangle\langle 1|$ as its final state. This state is resonantly driven towards the state $|3\rangle$, which is, however, strongly perturbed by the laser excitation of the $|2\rangle \leftrightarrow|3\rangle$ transition with Rabi frequency $\Omega_{2}$. This driving causes an AC Stark splitting of the state $|3\rangle$ by $\Omega_{2}$ and detunes the weak transition by $\Omega_{2} / 2$, which emerges as a generalized Rabi frequency of the weak and oscillatory amplitude transfer. The incoherent rate processes between states $|2\rangle$ and $|3\rangle$ damp these off-resonant oscillations on the time scale of a few $\left(\gamma_{2}\right)^{-1}$. The matrix $E(t)$ has not yet reached its steady state value, and despite the coherent coupling strength $\Omega_{1}$, it is only for times longer than the time scale $\left(\gamma_{1}\right)^{-1}$, that the correlation function regresses to the uncorrelated product. Within the picture of an evolving matrix $E(t)$, we find this suppression of coherent dynamics similar to the quantum Zeno dynamics [23], observed for evolving density matrices 24 .

\section{Conclusion}

Temporal correlation functions witness the non-classical character of light emitted from quantum sources, and they have played an immense role in the analysis and in the application of quantum states of light. In this article we have revisited the calculation of such correlation functions in terms of generalized measurements, and we have discussed how the correlations can be interpreted in terms of measurement back action and transient evolution of the emitter system quantum state during continuous probing.

Transients after discrete photon counting events have been well understood as the 
conventional state dynamics from the non-steady state prepared by the measurement back action of the counting event. To account for transients before such discrete measurement events, we employed the theory of past quantum states which allows calculation of measurement outcome probabilities, conditioned on the knowledge of both earlier and later measurements on the system. The practical calculations associated with this method are equivalent to the ones of the conventional master equation and the quantum regression theorem, but they deal explicitly with the transient evolution from a certain initial (final) state, according to an equation with enough similarities with the conventional master equation to offer insight into the dynamics.

We applied the method to amplitude-intensity correlations in the fluorescence from laser driven two- and three-level atoms. We recall, that the pertaining correlation functions have already been calculated in previous works, but without the interpretations offered by the present analysis.

\section{Acknowledgments}

This work was supported by the Villum Foundation and the IARPA MQCO program. EG acknowledges discussions with C. Navarrete-Benlloch and A. GonzalezTudela.

\section{References}

[1] Hanbury-Brown R and Twiss R Q 1956 Nature 17727

[2] Glauber R J 1963 Phys. Rev. 1302529

[3] Louisell W H 1973 Quantum Statistical Properties of Radiation (New York: John Wiley \& Sons, Inc.)

[4] Loudon R 1981 The Quantum Theory of Light (London: Oxford University Press)

[5] Cohen-Tannoudji C, Dupont-Roc J and Grynberg G 1992 Atom-Photon Interactions (New York: Wiley-Interscience)

[6] Gardiner C W and Zoller P 2000 Quantum Noise 2nd edn (Berlin: Springer-Verlag)

[7] Carmichael H J 1993 An Open System Approach to Quantum Optics (Lecture Notes in Physics, New Series m: Monographs, m18) (Berlin: Springer-Verlag)

[8] Gammelmark S, Julsgaard B and Mølmer K 2013 Phys. Rev. Lett. 111160401

[9] Kimble H J and Mandel L 1976 Phys. Rev. A 132123

[10] Carmichael H J 1999 Statistical Methods in Quantum Optics 1 (Berlin: Springer)

[11] Dalibard J, Castin Y and Mølmer K 1992 Phys. Rev. Lett. 68580

Mølmer K, Castin Y and Dalibard J 1993 J. Opt. Am. B 10524 (1993)

[12] Wiseman H M and Milburn G J 2010 Quantum Measurement and Control (New York: Cambridge University Press)

[13] Foster G T, Orozco L A, Castro-Beltran H M and Carmichael H J 2000 Phys. Rev. Lett. 853149

[14] Gerber S, Rotter D, Slodička L, Eschner J, Carmichael H J and Blatt R 2009 Phys. Rev. Lett. 102 183601

[15] Denisov A, Castro-Beltran H M and Carmichael H J 2002 Phys. Rev. Lett. 88243601

[16] Wiseman H M 2002 Phys. Rev. A 65032111

[17] Aharonov Y, Albert D Z and Vaidman L 1988 Phys. Rev. Lett. 601351

[18] Nielsen M and Chuang I 2000 Quantum Computation and Quantum Information (Cambridge: Cambridge University Press)

[19] Tan D, Weber S, Siddiqi I, Mølmer K and Murch K W 2015 Phys. Rev. Lett. 114090403

[20] Rybarczyk T, Gerlich S, Peaudecerf B, Penasa M, Julsgaard B, Mølmer K, Gleyzes S, Brune M, Raimond J -M, Haroche S and Dotsenko I 2014 arXiv:1409.0958 
[21] Carmichael H J, Castro-Beltran H M, Foster G T and Orozco L A 2000 Phys. Rev. Lett. 851855

[22] Marquina-Cruz E R and Castro-Beltran H M 2008 Laser Phys. 18157

[23] Facchi P, Nakazato H and Pascazio S 2001 Phys. Rev. Lett. 862699

[24] Shao X Q, Chen L, Zhang S and Yeon K -H 2009 J. Phys. B 42165507

Raimond J M, Sayrin C, Gleyzes S, Dotsenko I, Brune M, Haroche S, Facchi P and Pascazio S 2010 Phys. Rev. Lett. 105213601 